\title{
Process and Evaluation of Automated Robotic Fabrication System for In-Situ Structure Confinement
}

\author{
B. Bala Murali Kumar ${ }^{1}$, Yun Chung Hsueh ${ }^{1}$, Zhuoyang Xin ${ }^{1}$, and Dan $\operatorname{Luo}^{2(\bowtie)}$ \\ ${ }^{1}$ School of Civil Engineering, University of Queensland, Brisbane, Australia \\ ${ }^{2}$ School of Architecture, University of Queensland, Brisbane, Australia \\ d. luo@uq. edu. au
}

\begin{abstract}
The additive manufacturing process is gaining momentum in the construction industry with the rapid progression of large-scale 3D printed technologies. An established method of increasing the structural performance of concrete is by wrapping it with Fibre Reinforced Polymer (FRP). This paper proposes a novel additive process to fabricate a FRP formwork by dynamic layer winding of the FRP fabric with epoxy resin paired with an industrial scale robotic arm. A range of prototypes were fabricated to explore and study the fabrication parameters. Based on the systemic exploration, the limitations, the scope, and the feasibility of the proposed additive manufacturing method is studied for large scale customisable structural formworks.
\end{abstract}

Keywords: Robotic fabrication · Customisable formworks · FRP layer winding

\section{Introduction}

Recent studies on topology optimisation (Ning Gan 2021) have found that the material efficiency can be significantly improved by using irregular sections to replace the conventional sections in structural members. The optimized structures are also tended to be with changing cross-sections along the member span or height (Lloret Fritschi 2017), such as the tree-like structure used at the Qatar National Convention Centre and the Art Nouveau Apartment by Flying Concrete in San Miguel De Allende Mexico. The conventional concrete casting and steel manufacturing process is highly efficient and less time consuming for standardised cross sections but have their limitations on irregular shapes and sizes.

Additive Manufacturing (AM) on the other hand, is known for its flexibility and effectiveness for fabricating customised parts with complex geometries. AM techniques are used in various industries to create physical prototypes as well as manufacturing end parts. The construction industry has started to adopt the AM process and progressed from labs to printing full scale 3D structures (Souza 2020) in various parts of the world. The AM allows architects and engineers the geometrical freedom to produce highly efficient, non-standard building components without significant increase in cost and time. 
However, as additive manufacturing is different from common construction process, there has always been a challenge on how the additive manufacturing could be compatible with common structure system and standard assembly process in construction.

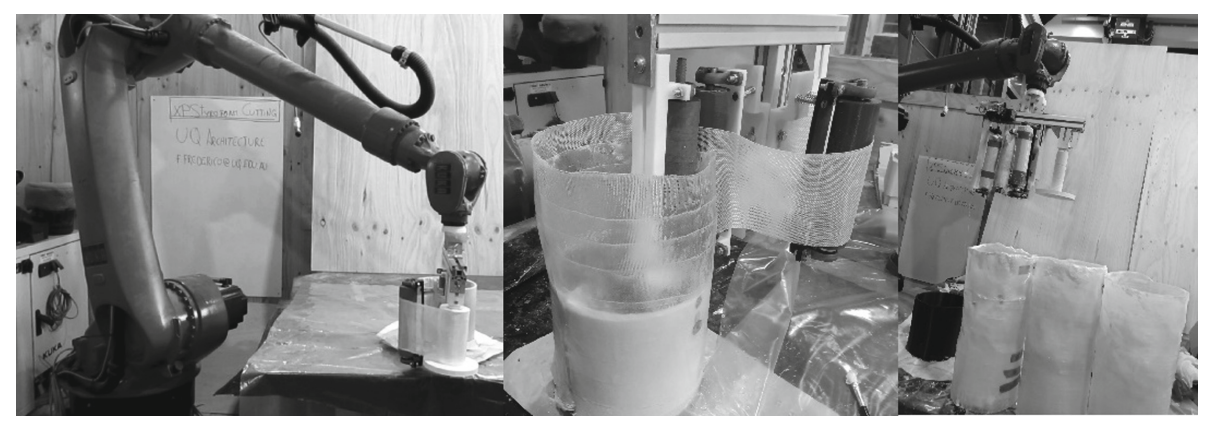

Fig. 1. Robotic fabrication.

Combining the concept of additive manufacturing and the existing established application of FRP in structure reinforcement and formwork, our research is introduced to explore the potential use of robotic technology for robotic fabrication as shown in Fig. 1 of structural members with greater formwork flexibility, and to increase the material and structural efficiency of the building structure.

\section{Background}

The various AM techniques can be broadly divided into seven categories (ISO/ASTM 2015): binder jetting, material extrusion, powder bed fusion, directed energy deposition, sheet lamination, vat photopolymerization and material jetting. The AM systems offers flexibilities in fabricated geometries with the degrees of flexibilities in the system. For example, the gantry robot has three degrees of freedom, systems based on industrial robotic arms have six degrees of freedom, which can be further expanded with a mobile base. Furthermore, these systems can also be enhanced with both terrestrial and aerial collaborative robots ((Lloret Fritschi 2017); (Keating 2017)).

The most prevalent AM method in construction is based on material extrusion, where a nozzle with fresh cementitious material is deposited along a horizontal layer in a predefined path. In the concrete extrusion process, the AM systems are categorized based on the layer thickness, size of the printed object, the printing environment, the assembling strategy, the use of support structures and the robot complexity. Several objects were implemented with these systems namely Curved Bench by Loughborough University (Lim et al. 2012), Complex Wall by XtreeE: (XtreeE), WinSun printed building components for the Dubai Future Foundation (Anon 2018).

Other innovative AM systems, such as the particle bed process FreeFAB, Smart Dynamic Casting and Mesh Mould increases the accuracy, ease the printing of complex shape and geometry of the architectural building components which are otherwise not possible using the traditional AM construction systems. The AM system called Smart 
Dynamic Casting by ETH Zuirch prints complex-shaped columns with changing cross sections. The formwork is printed, and the concrete extrusion process takes place simultaneously along a trajectory by a robot arm. The hydration process is monitored using sensors for the addition of admixtures and controlling the printing speed. In the mesh mould method, an In-situ fabricator which is an autonomous mobile robot bends and welds the steel to create a mesh. The mesh acts as a formwork and reinforcement for the concrete.

Current AM systems focuses on the traditional concrete structures which faces the challenges of expanding from small scale to large scale, and the integration of steel reinforcement into the system. Comparably, the large-scale additive manufacturing of FRP-concrete composites may have special advantage in resolving those issues.

\section{Fabrication Methodology}

\subsection{Robotic System Design - End Effector}

End effector is a customized robotic system developed for the FRP fabric weaving in additive manufacturing to create the desired formwork. The end effector design consists of two support rollers, the guide roller, and the FRP fabric support holder as shown in Fig. 2. The support rollers are mounted on a steel frame system linked with springs, which helps them to apply adequate pressure against each other and maintain their spatial position during the fabrication process. The FRP weave will be strategically positioned between these two rollers and the hollow formwork geometry could be maintained by the pressure as the rollers are passively driven. The guiding roller is responsible for the smooth transition from the FRP fabric roll to a straight fabric while maintaining the tension to prevent wrinkles in the additive layers. The dimensions of the end effector's rollers are $51.5 \mathrm{~mm}$ in diameter $\boldsymbol{d}, 200 \mathrm{~mm}$ in length $\boldsymbol{l}$, the inner mould radius $\boldsymbol{R} \boldsymbol{i}$ is 81 $\mathrm{mm}$ and the outer radius $\boldsymbol{R} \boldsymbol{o}$ is $329 \mathrm{~mm}$ as shown in Fig. 2 b.

\subsection{Material Parameters}

The commercially available FRP fabric, $150 \mathrm{~mm}$ in height, installed directly onto the support holder without any pre-processing requirement, is used for this additive manufacturing process. Every FRP fabric layer is required to bond with the preceding and succeeding layers to gain the formwork's strength and stiffness properties to resist against the concrete's hydrostatic pressure. This bonding action is achieved through the epoxy resin adhesives.

The epoxy resin was a two-part mixture of Amperg 22 with fast hardener mixed in a ratio 100:40 specified by the manufacturer. The working time for the epoxy resin is only $15 \mathrm{~min}$ beyond which the epoxy resin starts curing by exhibiting exothermic reactions. The curing time of the adhesive impregnated FRP fabric was different. The epoxy resin when applied to fibres, took approximately $90 \mathrm{~min}$ to $120 \mathrm{~min}$ to completely cure. However, it was found that when an external heating apparatus, such as use of a heating gun at $200 \mathrm{C}$, the curing time reduced to approximately $20 \mathrm{~min}$ for every 2.5 layers. 


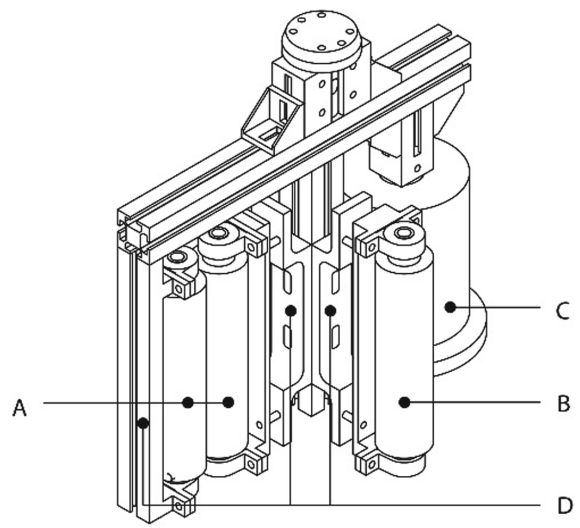

(a)

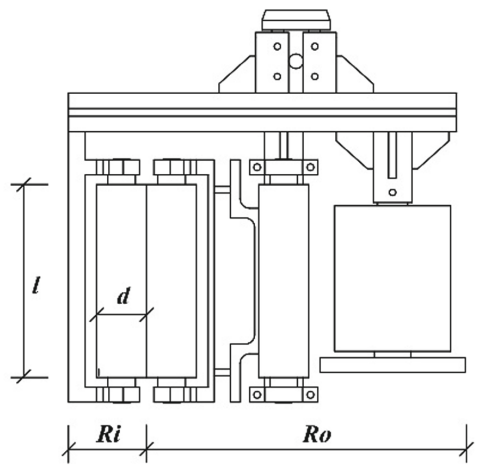

(b)

Fig. 2. (a) End effector design: A - the supporting rollers, B - the guiding roller, $\mathrm{C}-$ the FRP fabric holder, D - the steel frame system of the rollers. (b) Fundamental dimension of end effector design.

\subsection{Additive Fabrication Process}

The design stage of the additive process and the complex geometries are carried out in the Rhino/Grasshopper software environment. The surface of the geometry is extracted to plan the path planning algorithm for the robot's end effector to move along the designated path. To detect any potential crashes and deviations in the path logic, the algorithm is tested and visually inspected in the rhino environment. Next, the algorithm is given as an input to the KUKA robot plugin available in the grasshopper made by KUKA itself. The script is then generated by the plugin and copied into the manual controller of the KUKA robot to start the fabrication process. A flowchart of the fabrication process is shown in Fig. 3.

The additive manufacturing technique of the FRP formwork for different cross sections, shapes and non-linear geometries depends on the preceding layer's stiffness, strength, and torsional resistance. The first layer of the FRP formwork depends on an external support, termed "base mould", to serve as the foundation for succeeding layers. The base mould is 3D printed with polylactic acid plastic (PLA), and statically bolted to the fabrication table to prevent any lateral or slip movement during the fabrication process. The dimension of the base mould used in this paper is shown in Fig. 4b.

The end effector is then installed onto the robot's sixth axis hand and tested. The fabrication process follows a spiral path and hence a data configuration file was set for the robot's sixth arm to rotate more than \pm 360 degree in a limitless spindle manner. The end effector is calibrated with the mould spatial location with respective to KUKA robot's location in the 3D space.

From experience, it is suggested that the generated path planning script is verified by allowing the robot to run without any fabric and epoxy resin. After the successful completion of the dry run, the experimental preparation and precaution measures against epoxy resin is elaborated in the following section. The end effector is lowered to the bottom of mould and fabric is attached with the mould. The epoxy resin is prepared 




Fig. 3. Fabrication process

in a mix ratio of 100:40 as per manufacturer's specification and applied on the fabric as described in the previous section. An external heat source is applied for a particular number of revolutions up to the calibrated durations. In this experiment, a heat gun was used for a duration of $30 \mathrm{~min}$ for every 2.5-layer revolution. The layer winding, preparation and application of epoxy resin on the fabric and heat application are repeated until the desired fabrication height is achieved as shown in Fig. 4a. This fabricated FRP formwork is then demoulded from the base mould by disconnecting the initial mould to fabric connections. A time lapse of the formwork fabrication process is shown in Fig. 5.

\subsection{Parameters Influencing the Additive Process}

The potential parameters that have an influence on the additive manufacturing process that determines the stiffness, strength, flexibility, and feasibility for the large-scale process are summarised as follows.

\subsubsection{Epoxy Curing Parameters}

The adhesive property of the epoxy resin is achieved after curing. The curing time is influenced by two major factors: temperature and number of layers. Temperature above $200{ }^{\circ} \mathrm{C}$ can damage the FRP fabric. Therefore, care must be taken to not apply the 


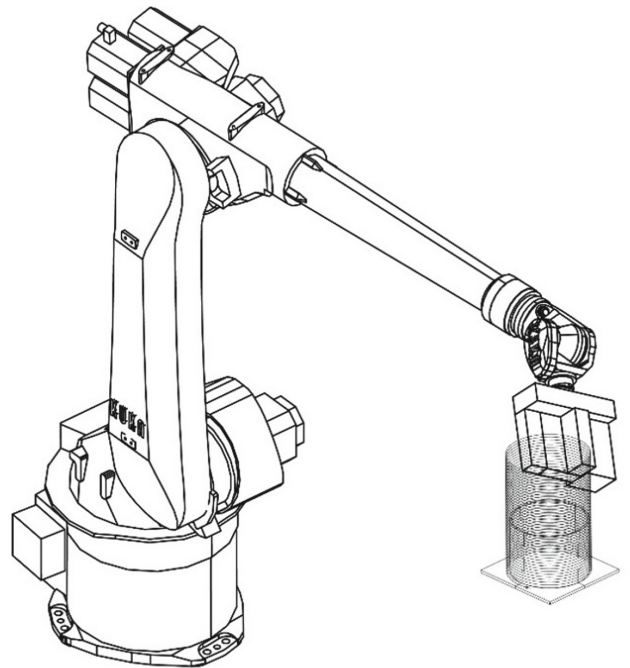

(a)



(b)

Fig. 4. (a) The robotic fabrication system. (b) The base mould.

temperature at an area for a prolonged time to avoid FRP fabric damage. In addition to the temperature magnitude, the curing time is also influenced by the number of layers that is subjected to the heat source at a given time. A number of trials were conducted to find the optimum temperature without damaging the layers and number of layers to determine the curing time. In this paper, all the prototypes were subjected to a temperature of $200{ }^{\circ} \mathrm{C}$ at every 2.5 revolutions for a period of $30 \mathrm{~min}$. The external heat source used to apply the temperature in this paper was a heat gun while other possible curing methodology such as UV enabled resin curing will be explored in future. The waiting time to manually apply the epoxy resin was set to $20 \mathrm{~s}$ for every $1 / 8^{\text {th }}$ of the circumference of the cross section.

\subsubsection{Fabrication Time Estimation}

The theoretical time required to complete the prototype fabrication can be estimated using Eqs. (1), (2) and (3) shown below. The total theoretical time depends on the height of the prototype geometry, the height of the FRP layer, the number of overlap layers, the time required to apply the epoxy resin and the FRP winding process. The total time shown here is an estimate but the experimental time may be different due to large environmental factors influencing the fabrication process.

$$
\begin{gathered}
\mathrm{t}_{\text {total }}=\frac{H n_{O L}}{h_{f r p}}\left(t_{1}+t_{2}\right) \\
t_{1}=\frac{\pi d}{V_{\text {winding }}} \\
t_{2}=n_{\text {resin }} t_{\text {resin }}
\end{gathered}
$$




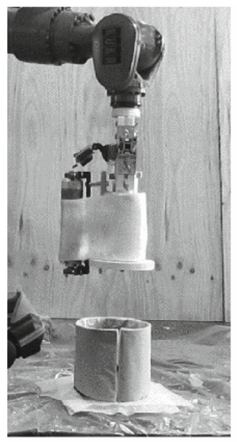

(a)

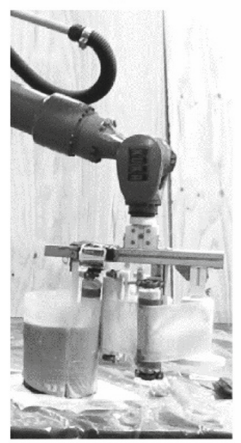

(e)

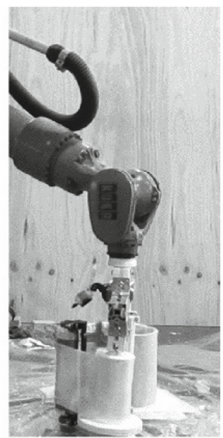

(b)

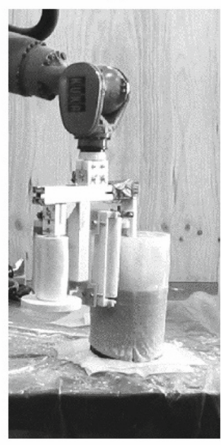

(f)

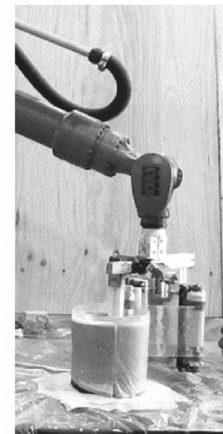

(c)

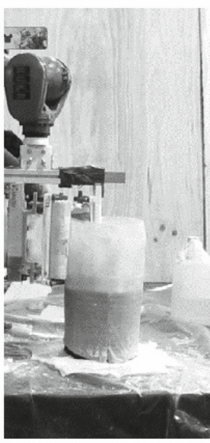

(g)



(d)

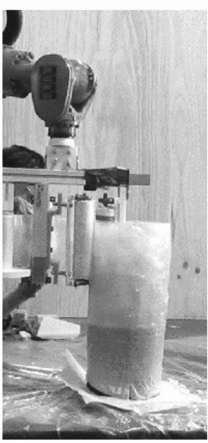

(h)

Fig. 5. Time lapse of FRP formwork fabrication.

$\mathrm{t}_{\text {total }}$ - the estimated total time for prototype fabrication, $H$ - the total height of the designed prototype, $n_{O L}-$ the maximum layer overlap number, $h_{f r p}-$ the height of one-layer FRP fabric, $t_{1}$ - the robotic winding time for each circumference motion, $t_{2}$ - the epoxy resin applying time for each circumference motion, $d$ - the diameter of the hollow cylinder section, $V_{\text {winding }}-$ the constant winding velocity, $n_{\text {resin }}-$ the times of resin applying for each circumference motion, $t_{\text {resin }}$ - one epoxy resin applying time.

\subsubsection{Fabrication Speed}

The fabrication speed of the additive process depends on factors such as curing time, layer winding speed and area of the column covered by the end effector in a single revolution. The fabrication speed parameter is optimized between the minimum time required to complete the additive process and the quality of the structural component. The quality of the structural component is the deviation between the cross section of the design and the additive component. In this paper, the layer winding speed was set to $1 \mathrm{~cm} / \mathrm{s}$ and curing time for every 2.5 layers was $30 \mathrm{~min}$ at $200{ }^{\circ} \mathrm{C}$. 


\section{Results and Discussion}

A preliminary study was conducted to assess the feasibility and flexibility of proposed novel additive process for large-scale additive production of architectural elements. Initially, two trial experiments were conducted as shown in Fig. 6 (A)-(B) to calibrate the end effector, epoxy resin mixture to determine the optimum number of layer revolutions application, estimated time for each of the operation and the Rhino/grasshopper script as summarised in Table 1. The experience gathered from the two trials paved the way to establish a procedure for the future additive process for the prototypes is shown in Table 2. After the initial trial experiments, three preliminary prototypes were fabricated as shown in Fig. 6 (C)-(E) to study the challenges and issues associated with the proposed additive process. The objective of these preliminary study is to identify the parameters and challenges that can be controlled or solved to construct the proposed additive technique feasible for large-scale additive process of architectural elements.

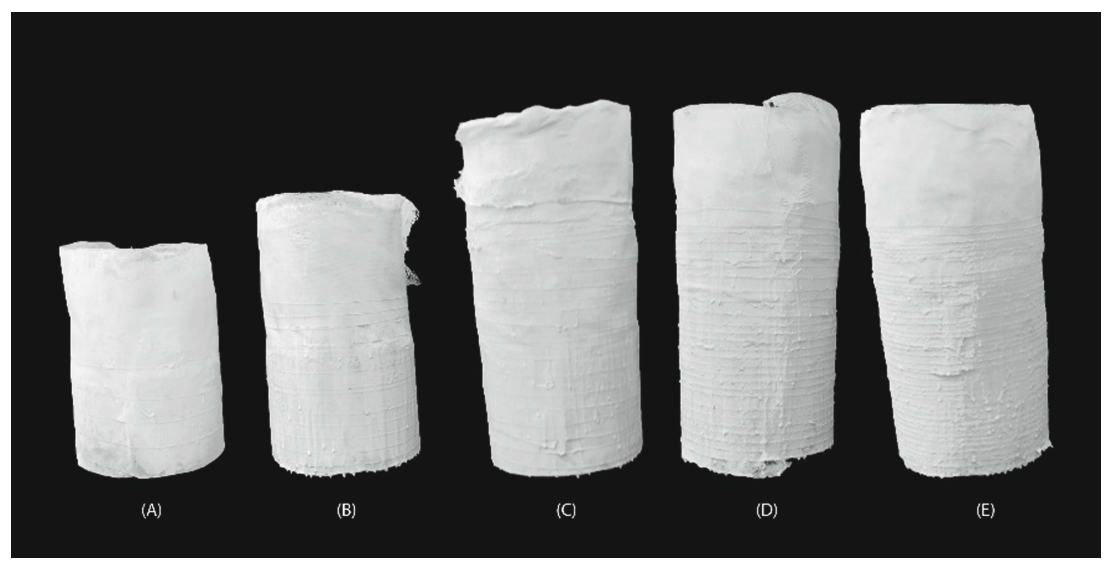

Fig. 6. The FRP formworks - (A) trial 1 (B) trial 2 (C) 10 layers (D) 15 layers (E) 20 layers

\subsection{Accuracy of the Proposed Additive Process}

The physical environmental factors play a key role in determining the accuracy of the fabrication of the component. For the prototype to be compared fairly with the additive process and not wrapping on the base mould, the entire prototype was subdivided into three segments where the middle segment is $150 \mathrm{~mm}$ tall.

The accuracy between the digital design and the additive process is compared by $3 \mathrm{D}$ scanning the fabricated prototype. The $3 \mathrm{D}$ scanning process is used to extract the surface details in the form of three-dimension spatial coordinate points. These points are meshed together to form the object geometry in digital design space. During the 3D scanning process, the probability of collecting data points other than our prototype due to the environmental factors can affect our accuracy estimation. Therefore, the data is pre-processed by setting the threshold value of the data points to a maximum radius of 10 
Table 1. Estimated time analysis for the prototypes for 2.5-layer additive process

\begin{tabular}{l|l|l|l|l}
\hline \multirow{2}{*}{ No } & Work & Time & \multicolumn{2}{l}{} \\
\cline { 2 - 5 } & Trials 1 and Trial 2 & $\begin{array}{l}10 \text { Layers/h } \\
(\mathrm{s})\end{array}$ & $\begin{array}{l}5 \text { Layers/h } \\
(\mathrm{s})\end{array}$ & 2.5 Layers/h (s) \\
\hline 1 & Mixing epoxy resin & 60 & 60 & 60 \\
\hline 2 & Resin apply + wait & 10 & 20 & 40 \\
\hline 3 & Wrap 45 degree & 5 & 10 & 20 \\
\hline 4 & Resin apply & 10 & 20 & 40 \\
\hline 5 & Wrap 45 degree & 5 & 10 & 20 \\
\hline & Repeat steps from 1 to 5 until the revolution of 2.5 additive layers \\
\hline 6 & Heating gun drying & 600 & 1200 & 2400 \\
\hline $2-6$ & Estimated time/ 2.5additive layer & $0.25 \mathrm{~h}$ & $0.5 \mathrm{~h}$ & $1 \mathrm{~h}$ \\
\hline
\end{tabular}

Table 2. Trials and prototypes performed for the experimental study

\begin{tabular}{l|l|l|l|l|l}
\hline S.No & Prototype & Height $(\mathrm{mm})$ & Number of layers & $\begin{array}{l}\text { Approximate } \\
\text { fabrication time } \\
\text { (hours) }\end{array}$ & $\begin{array}{l}\text { Layer winding } \\
\text { speed }(\mathrm{cm} / \mathrm{s})\end{array}$ \\
\hline 1 & Trial 1 & 300 & 5 & 3 & 1 \\
\hline 2 & Trial 2 & 400 & 10 & 5 & 1 \\
\hline 3 & 10 layer & 450 & 10 & 6 & 1 \\
\hline 4 & 15 layer & 450 & 15 & 10 & 1 \\
\hline 5 & 20 layer & 450 & 20 & 14 & 1 \\
\hline
\end{tabular}

mm ensuring the collected data points represent only the prototype. The design geometry and the meshed geometry are then overlapped for comparison purposes. The coordinates of the data points are then averaged over a thickness axis to obtain the average thickness of the prototypes.

With the average thickness estimated in the spatial coordinate space, the deviation of the additive process in fabricating the prototype as designed in the software can be compared. The comparison of three prototypes for the three different number of layers of 10, 15 and 20 can be compared. It can be observed from Fig. 7 that the accuracy of the scanning process is approximately $97 \%, 98 \%$ and $98 \%$ for 10,15 , and 20 layers respectively. The comparison between the $3 \mathrm{D}$ scan and the digital design in shown distinctively in (b)-(d) and (e) indicates the measurements of the prototypes section to calculate the average deviation. 


\begin{tabular}{|c|c|c|c|c|c|c|c|}
\hline Layer & $\begin{array}{l}\text { Average } \\
\text { Deviation }\end{array}$ & Accuracy & $\begin{array}{l}\text { (a) Physical } \\
\text { Prototype }\end{array}$ & (b) 3D Scan & $\begin{array}{l}\text { (c) Designed Geo and } \\
\text { 3D Scan Overlap }\end{array}$ & $\begin{array}{l}\text { (d) Measurement } \\
\text { Strip }\end{array}$ & $\begin{array}{l}\text { (e) Deviation } \\
\text { Range }\end{array}$ \\
\hline 10 & $3.58 \mathrm{~mm}$ & $96.9 \%$ &  & & & & \\
\hline 15 & $3.57 \mathrm{~mm}$ & $98.4 \%$ & 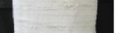 & & & & \\
\hline 20 & $3.53 \mathrm{~mm}$ & $97.8 \%$ & & & & & \\
\hline \multicolumn{3}{|c|}{ with threshold value $c=10.0 \mathrm{~mm}$} & & \multicolumn{4}{|c|}{ "+" for inside the designed shape, "-" for outside the designed shape } \\
\hline
\end{tabular}

Fig. 7. Accuracy comparison between the physical prototype and rhino design

\subsection{Discussion}

The Fabrication system proposed in this research is an integration of the emerging technologies of advanced manufacturing with architectural design and structural engineering. This system is designed for the rapid fabrication of topological optimized-based generative form with customizable FRP formwork. The major parameters that influenced the proposed additive process were identified and investigated. In this research, parameters regarding the selection/designs of epoxy resin, end effector, base mould and the FRP fabric were investigated during the additive fabrication of the prototypes. The increase in layer winding speed and the number of layers can greatly influence the total fabrication time of the prototypes. From the observation, it is imperative to seek other alternate source of FRP fabric adhesion, alternate ways to increase the temperature of the epoxy resin to lower the fabrication time.

From our current study, it is evident that the proposed additive process has the potential to bring a change in the fabricated structure component because of the strength, design, geometrical flexibility and cost effectiveness, while dealing with the following disadvantages like the high fabrication time, manual epoxy resin application. Thus, further investigation of the methodology and technique to implement for large scale non-uniform and complex geometry by integrating the FRP and concrete is required. 


\section{Conclusion}

The additive manufacturing process in the industry is gaining momentum. Though there are rapid development in 3D concrete printing, from the perspective of structural engineering, we require the material and cost-effective system that is compatible with current construction process. This paper explores the feasibility of using the additive process of FRP dynamic winding of formwork for concrete casting of customizable structure components in large scale construction. The additive process proposed in this paper uses an FRP that is operated by KUKA robot with customized end effector, and the epoxy resin is used as the adhesive for lamination in the process. Three prototypes and two trial experiments were conducted to study the influencing parameters and record the deviation of the fabricated components comparing with the original design. From the preliminary studies, it can be concluded that there is only less than $4 \%$ deviation between the fabricated components and the digital model, yet further improvements in subsequent experiments is highly likely. Thus, the proposed additive process has the potential to manufacture fabricated large-scale structural components of topologically optimized shape with irregular cross section and non-uniform geometry.

\section{References}

Anon: 3D Printing with concrete: state-of-the art, 2018(4), 275-287 (2018)

Bao, D.W.: Bioinspired generative architectural design formfinding and advanced robotic fabrication based on structural performance. In: 1st International Conference on Computational Design and Robotic Fabrication (CDRF 2019), pp. 147-170 (2019)

Lloret Fritschi, E., et al.: Smart dynamic casting, slip forming with flexible formwork-inline measurement and control. In: Norway, Proceedings of the 11th High Performance Concrete (11th HPC) and the 2nd Concrete Innovation Conference (2nd CIC) (2017)

ISO/ASTM: ISO/ASTM 52900:2015 Additive manufacturing — General principles — Terminology (2015)

Keating, S.J., Leland, J.C., Cai, L., Oxman, N.: Toward site-specific and self-sufficient robotic fabrication on architectural scales. Sci. Robot. 2(5), eaam8986 (2017). https://doi.org/10.1126/ scirobotics.aam8986

Souza, M.T., Ferreira, I.M., de Moraes, E.G., Senff, L., de Oliveira, A.P.N.: 3D printed concrete for largescale buildings: an overview of rheology, printing parameters, chemical admixtures, reinforcements, and economic and environmental prospects. J. Build. Eng. 32, 101833 (2020)

Ning Gan, Q.W.: Topology optimization of multiphase materials with dynamic and static characteristics by BESO method. Adv. Eng. Softw. 151, 102928 (2021)

Lim, S., et al.: Developments in construction-scale additive manufacturing processes. Autom. Constr. 21(1), 262-268 (2012)

XtreeE: XtreeE. http://www.xtreee.eu/. Accessed 24 June 2019 
Open Access This chapter is licensed under the terms of the Creative Commons Attribution 4.0 International License (http://creativecommons.org/licenses/by/4.0/), which permits use, sharing, adaptation, distribution and reproduction in any medium or format, as long as you give appropriate credit to the original author(s) and the source, provide a link to the Creative Commons license and indicate if changes were made.

The images or other third party material in this chapter are included in the chapter's Creative Commons license, unless indicated otherwise in a credit line to the material. If material is not included in the chapter's Creative Commons license and your intended use is not permitted by statutory regulation or exceeds the permitted use, you will need to obtain permission directly from the copyright holder.

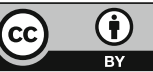

